\title{
MAPEAMENTO DOS FRAGMENTOS FLORESTAIS DE PROSOPIS JULIFLORA A PARTIR DE SENSORIAMENTO REMOTO: SUBSÍDIO AOS ESTUDOS DE SUAS IMPLICAÇÕES AMBIENTAIS NO BIOMA CAATINGA NO NORTE DA BAHIA
}

\author{
Nerivaldo Afonso dos Santos ${ }^{(a)}$, Philipe Damasceno Pedreira ${ }^{(b)}$ Kezia Andrade dos Santos ${ }^{(c)}$ \\ (a) Departamento de Ciências Humanas e Filosofia, Universidade Estadual de Feira de Santana, Email: \\ nerivaldo.geo@gmail.com \\ (b) DCHF, UEFS, Email: philipe_pedreira@ @otmail.com \\ (c) DCHF, UEFS, Email: kezia_peniel@ @otmail.com
}

\section{Eixo: GEOTECNOLOGIAS E MODELAGEM ESPACIAL EM GEOGRAFIA FÍSICA}

\begin{abstract}
RESUMO
Na região semiárida vários são os fatores que causam degradação do meio ambiente, dentre estes: variações meteorológicas, degradação dos solos com superpastoreio, práticas de manejo irregular, perda da cobertura vegetal. No Nordeste brasileiro, onde está inserido o bioma Caatinga, esses fatores de pressão atuam de maneira intensa sobre os ecossistemas, outro fator de preocupação é a inserção de espécies vegetacionais com características de monodominância em áreas de vegetação nativa, a expansão dessas espécies caracteriza-se como invasão biológica, pondo em risco a biodiversidade do bioma local. O presente trabalho objetivou mapear as áreas de ocorrência de Algarobas (Prosopis juliflora (SW) DC), no Polo de Jeremoabo-BA, realizou-se o mapeamento através do algoritmo de classificação Spectral Angle Mapper (SAM) utilizando como membro de referência curvas espectro-temporais de Enhanced Vegetation Index (EVI) do sensor Moderate Resolution Imaging Spectroradiometer (MODIS), esses dados compõe uma série temporal dos anos de 2001 a 2012, totalizando 276 imagens.
\end{abstract}

Palavras chave: Séries temporais; Indicador ambiental; Semiárido, Desertificação Introdução

A inserção da algaroba na região semiárida do Brasil ocorreu por meio de política governamental, cuja principal ideia disseminada foi o alto potencial forrageiro (GOMES, 1961). A origem da planta é peruana e as primeiras plantações, com apenas quatro árvores, decorreram no estado de Pernambuco, em 1942. Posteriormente houve uma disseminação da algaroba para demais estados, devido a adaptação da planta às condições ambientais semiáridas brasileiras e sua utilidade na dieta animal. As primeiras translocações de espécies de uma região a outra do planeta foram intencionais e buscavam, basicamente, suprir necessidades agrícolas, florestais e outras de uso direto (LEÃO et al., 2011), assim como ocorreu com a algaroba.

A característica destacável do nordeste semiárido é, sem dúvida, as feições vegetais da caatinga (AB'SABER, 2003), não diferindo para os municípios que compõem o Polo de Jeremoabo. Nesse, a vegetação é constituída de espécies deciduais e semideciduais, com porte arbóreo, arbustivo, de aspecto rústico (AB'SABER, 2003). 


\section{OS DESAFIOS DA GEOGRAFIA FÍSICA NA FRONTEIRA DO CONHECIMENTO Instituto de Geociências - Unicamp Campinas - SP \\ 28 de Junho à 02 de Julho de 2017}

No semiárido da Bahia, averiguou-se a intensa propagação das matas de algaroba nos municípios que compõem o Polo de Jeremoabo, onde predomina o clima tropical semiárido, com precipitação média anual de 300 a $500 \mathrm{~mm}$. O estudo em desdobramento é realizado em áreas com estágios avançados de degradação, o que lhe confere caráter de relevância no âmbito das discussões sobre desertificação, a planta algaroba foi introduzida no semiárido Nordestino como alternativa, devido ao seu potencial forrageiro para os animais, lenha, e até alimentação humana, porém estudos tem denotado certa preocupação, devido a sua capacidade de proliferação, ocasionando grandes perdas econômicas ou até mesmo biológica, pela extinção da biota nativa. As informações extraídas permitiram quantificar que as áreas mapeadas, as manchas de algarobas em sua maioria foram encontradas em altitudes que variam 244 a $430 \mathrm{~m}$.

Este estudo objetivou mapear as áreas de ocorrências de matas de algaroba no Pólo de Jeremoabo BA, através de séries temporais de imagens de satélite do sensor Modis (Moderate Resolution Imaging Spectroradiometer), visando subsidiar os estudos sobre os impactos e a gestão ambiental na área de estudo. O sensor MODIS é um dos principais instrumentos acoplados a plataforma TERRA e AQUA, do Sistema de Observação da Terra EOS (Earth Observing System) da NASA (National Aeronautics and Space Administration), sendo que dos cinco sensores abordo da referida plataforma seus produtos são os que mais têm se difundido entre pesquisadores, por apresentar em alta resolução temporal de 1 a 2 dias e composições a cada 8 ou 16 dias, com resolução radiométrica de 12 bits e 36 bandas espectrais, possui resolução espacial de $250 \mathrm{~m}$ nas duas bandas, $500 \mathrm{~m}$ em cinco outras bandas e $1000 \mathrm{~m}$ nas demais 29 bandas e um campo de visada de $2330 \mathrm{~km}$ de largura. (SOARES et al., 2007).

\section{Procedimentos metodológicos}

A área de estudo compreende o Polo Regional de Jeremoabo (Figura 1), localizada no extremo norte da Bahia, composto por 13 municípios: Antas, Novo Triunfo, Chorrochó, Rodelas, Uauá, Paulo Afonso, Glória, Pedro Alexandre, Coronel João de Sá, Macururé, Santa Brígida, Canudos e Jeremoabo. O polo está situado em uma região predominantemente escassa de precipitações, possui porção significativa de sua área caracterizada pelo clima árido e sem nenhum excedente hídrico (SEI 1999). Sendo uma das características marcantes do domínio morfoclimático da caatinga, no qual o Polo de Jeremoabo está inserido, a semiaridez climática (AB'SABER, 2003). 




Figura 1: Mapa de localização da área de estudo

Para o desenvolvimento dessa pesquisa, inicialmente foi realizada a revisão bibliográfica a fim de se obter embasamento teórico acerca da temática abordada, bem como as principais aplicações dos dados utilizados no desenvolvimento desta pesquisa e os conceitos mais relevantes discutidos, tais como séries temporais de índice de vegetação, precipitação e sua influência na dinâmica de classes de cobertura vegetal, áreas agrícolas, as influências desses fatores na degradação das terras. Para facilitar o entendimento da metodologia adotada nesta pesquisa o fluxograma a seguir (Figura 2) ilustra as principais etapas. 




Figura 2: Fluxograma com os principais procedimentos metodológicos utilizados na pesquisa.

Para este estudo adiquiriram-se imagens MODIS/Terra, produto MOD13Q1 (Vegetation Índices 16Day L3 Global 250 m) que contém os índices de vegetação EVI (Enhanced Vegetation Index), através do website (http://www.modis.cnptia.embrapa.br/geonetwork/srv/pt/main.home). Os dados utilizados foram disponibilizados pela Embrapa Informática e Agropecuária, com a proposta de facilitar ao usuário o acesso aos produtos MODIS, em recortes estaduais, as características dos dados são: formato GeoTIFF, Projeção Geográfica, Datum WGS-1984, resolução espacial de 250 metros, resolução temporal de 16 dias, com cenas compostas por pixels de valor máximo desse período. Foram adquiridas para este estudo um total de 276 imagens para um período compreendido entre 2001 a 2012, sendo 23 imagens/ano.

O EVI (Eq. 1), proposto por Huete et al. (2002), foi desenvolvido como alternativa para minimizar problemas de saturação por meio dos efeitos da atmosfera e do solo (JUSTICE et al., 1998).

$$
E V I=2,5 \frac{\rho_{I V P}-\rho_{V M}}{\rho_{I V P}+\left(6 \rho_{V M}-7,5 \rho_{A Z}\right)+1}
$$

onde: $\square_{A Z}$ corresponde à reflectância na banda espectral do azul (Modis banda 3), $\square_{V M}$ corresponde às reflectância na banda espectral do vermelho (Modis banda 1) e $\square_{I V P}$ corresponde à reflectância na banda espectral do infravermelho próximo (Modis banda 2). 




As séries temporais são afetadas pelos ruídos provenientes das condições meteorológicas, tais como chuvas, nuvens, sombra de nuvens, entre outros. Tais problemas são minimizados com a utilização de filtros, os quais promovem a suavização das curvas temporais. Neste trabalho utilizou-se o método Logística Dupla implementado no software Timesat (JÖNSSON e EKLUNDH, 2004) para filtragem dos dados. Esses dados foram recortados no software ENVI segundo os limites do Polo de Jeremoabo, organizados de maneira sequencial (cronológica) onde cada imagem foi nomeada com respectivos números que a identifique no tempo, compondo a série temporal.

Para se obter os espectros temporais de EVI, as imagens relativas às diferentes datas foram unidas, sintetizadas em um cubo 3D, de forma que a informação multitemporal pudesse ser extraída. Desta maneira, cada imagem utilizada na série temporal se refere a um intervalo de tempo na área de estudo e não, como tradicionalmente, a um intervalo do espectro da radiação eletromagnética (SAMPAIO, 2007). Os espectros-temporais foram organizados em sequência dos dias dos anos em ordem crescente, assim essa sequência representa a fusão do elemento tempo para a área de estudo, resultado da junção das imagens MODIS.

A distribuição das manchas de algaroba no Polo de Jeremoabo foi mapeada utilizando o Algoritmo SAM (Spectral Angle Mapper), implementado no software ENVI 5.0. O SAM utiliza espectros de referência para identificar pixels nas imagens que apresentam comportamento espectral semelhante. $\mathrm{O}$ classificador SAM rotula pixels pela similaridade angular entre o vetor padrão para uma determinada classe e o vetor de um pixel candidato (KRUSE et al., 1993), de maneira que quanto menor o ângulo, maior a similaridade espectral, enquanto que maior o ângulo, mais distintas são as classes. Neste trabalho foram testados diversos valores de ângulo para o algoritmo SAM, sendo adotado o valor 0,001. A figura 3 exemplifica o espectro temporal de pixels representativos de manchas de algaroba, construído a partir da biblioteca espectral utilizada como membro de referência para a classificação.

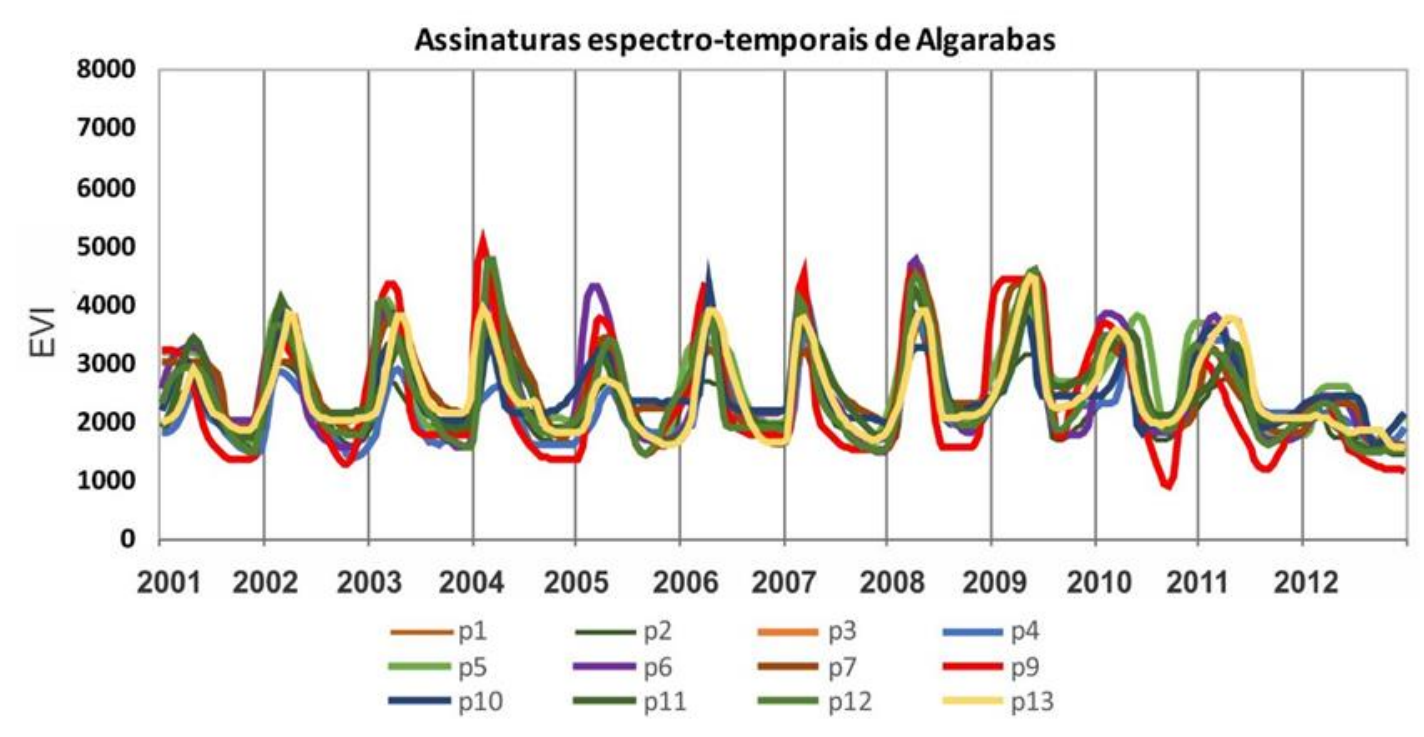


XVII Simpósio Brasileiro

de Geografia Fisica Aplicada

I Congresso Nacional

de Geografia Física

\section{OS DESAFIOS DA GEOGRAFIA FÍSICA NA FRONTEIRA DO CONHECIMENTO \\ Instituto de Geociências - Unicamp \\ Campinas - SP \\ 28 de Junho à 02 de Julho de 2017}

Figura 3: Assinaturas espectro-temporais de EVI representativas de Algarobas no período de 2001 a 2012.

No que se refere as assinaturas coletadas, como elas são representativas das algarobas esperava-se encontrar maior semelhança entre as curvas, haja vista que se tratava do mesmo alvo. Todavia, o fato de se ter manchas de algarobas em diferentes estágios vegetativos, em função destes dados representarem, não apenas a resposta espectral, mas também temporal, foram encontradas assinaturas com comportamentos distintos. Isto implicou no valor do ângulo estabelecido para o classificador SAM. A última etapa deste trabalho consistiu na validação dos resultados por meio da construção da matriz de confusão e da estatística kappa (LANDIS e KOCH, 1977).

\section{Resultados e discussão}

A partir do mapeamento da distribuição da algaroba no Polo de Jeremoabo pôde-se perceber que as manchas seguem um padrão de dispersão, ocorrendo em áreas planas (variando de $3^{\circ}$ a $6^{\circ}$ de declividade), em geral seguindo o curso de rios e drenagens, (Figura 3). Para demonstrar essa distribuição utilizou-se o modelo digital de elevação GDEM (Global Digital Elevation Model), (Figura 4). A imagem foi processada em ambiente SIG e as curvas de nível foram extraídas com intervalos de 30 metros para caracterização da variação de altitude na área de estudo. Posteriormente foi produzido um modelo digital de elevação TIN (Triangulated Irregular Network). A sobreposição deste produto do relevo com as áreas mapeadas com algaroba possibilitou a melhor visualização na variação de perfil do terreno em sua totalidade. Os valores de altitude variaram entre 150 a $900 \mathrm{~m}$.

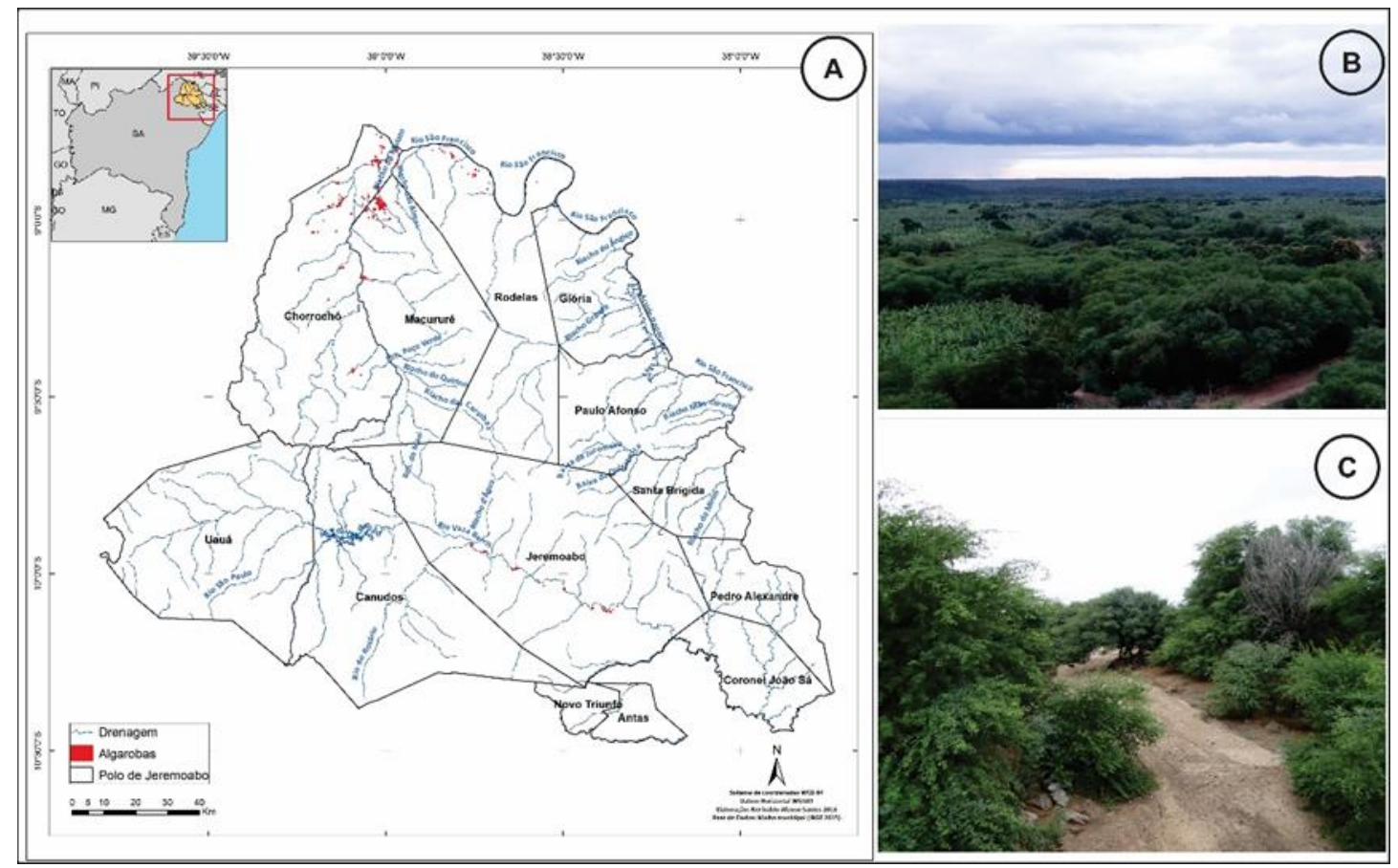


XVII Simpósio Brasileiro

de Geografia Fisica Aplicada

I Congresso Nacional

de Geografia Física

\section{OS DESAFIOS DA GEOGRAFIA FÍSICA NA FRONTEIRA DO CONHECIMENTO \\ Instituto de Geociências - Unicamp \\ Campinas - SP \\ 28 de Junho à 02 de Julho de 2017}

Figura 3. Mapa de rede de drenagem (a), fotografias de campo de áreas com monodominância de algaroba em proximidades de drenagens incipientes (b e c).

Segundo Pegado et al., (2006) a espécie também é frequente invasora em matas ciliares e baixadas sedimentares. Desse modo, a ocupação e a expansão da algaroba são bastante favorecidas pelas perturbações antrópicas e pela proximidade de cursos de água (LINS E SILVA, 1997).

No Nordeste, a algaroba ocorre preferencialmente em locais de clima semiárido, sendo encontrada em densos povoamentos de áreas degradadas, agrícolas e de pasto, como também ao longo de canais de irrigação e nas proximidades de açudes (LIMA et al., 2002).

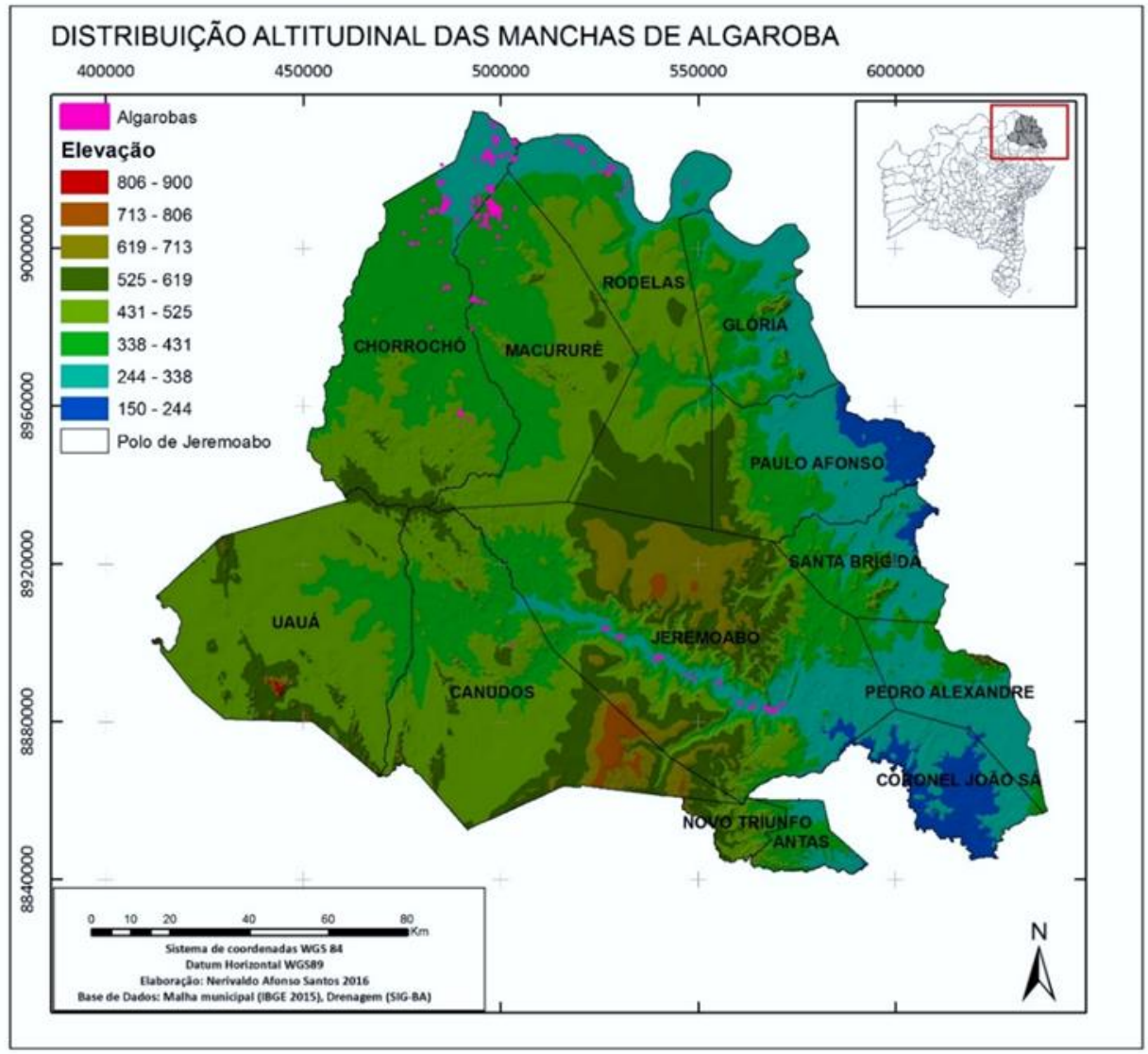

Figura 4. Distribuição das manchas de algarobas, associadas as classes altimétricas.

De acordo com os resultados, as manchas de algarobas não foram encontradas nas classes 1 (150 a 243 $\mathrm{m})$; na área que corresponde à classe $2(244-388 \mathrm{~m})$ manchas maiores que $80.000 \mathrm{~m} 2$ foram encontradas; para altitudes superiores a $430 \mathrm{~m}$ não foram registradas ocorrências de algaroba. As áreas mapeadas totalizaram cerca de 45,184 Km2. 




A expansão da algaroba como espécie invasora pode estar relacionada com a produção massiva de sementes e a sua eficiente dispersão. As sementes podem ser dispersas a longas distâncias. Em escala local, são os animais, tanto de criação quanto os selvagens, que disseminam as sementes após comerem os frutos. Outro fator que contribui para o sucesso da invasão da espécie é a capacidade da semente de permanecer dormente por até 10 anos no solo (GISP, 2005).

Para verificação da acurácia do mapeamento foram selecionados aleatoriamente 60 pontos no mapa de distribuição das algarobas, estes pontos foram analisados por meio de dados de campo e imagens de satélite de alta resolução. A estatística Kappa foi utilizada para validar os resultados encontrados. A Tabela 1 mostra a matriz de confusão, do total de 60 pontos, 47 foram classificados corretamente, resultando em uma exatidão global de 78\%, e o Índice Kappa foi de 0,47.

Tabela 1 - Matriz de confusão do mapeamento da distribuição da Algaroba

\begin{tabular}{cccc}
\hline & \multicolumn{3}{c}{ Classificação } \\
\hline & AL & N AL & \%EC \\
\hline AL & 37 & 2 & 9,7 \\
\hline N AL & 11 & 10 & 90,3 \\
\%EO & 22 & 78 &
\end{tabular}

$A L=$ Algaroba; $\mathbf{N} \overline{A L}=$ Não algaroba; $E C=$ Erro de comissão; $E O=$ Erro de omissão.

Os resultados obtidos podem ter sido influenciados pela baixa ocorrência de fragmentos de mata de algaroba menores que a resolução espacial dos dados MODIS de $250 \mathrm{~m}$. Como mata nativa e algaroba são classes que apresentam características espectrais muito semelhantes. Segundo OLIVEIRA, Et al., 2013, os erros de comissão ocorrem quando se incluem pixels em uma classe quando ele pertence a outra classe, e os erros de omissão acontecem quando são atribuídos a determinada classe os erros de classificação das outras classes.

\section{Considerações finais}

O mapeamento das áreas de algarobas no Polo de Jeremoabo, utilizando séries temporais de EVI e o classificador SAM permitiu conhecer a distribuição espacial e altimétrica dessas matas, e consequentemente, os locais onde essas matas se desenvolvem com maior expansão e podem causar impactos à vegetação nativa. Assim, é possível desenvolver estudos futuros para entender quais impactos são causados pela proliferação desta planta e quais medidas devem ser adotadas no plano de manejo, para que essas formações não afetem a dinâmica do ecossistema local. Desta forma, esse 
estudo se apresenta como subsídio à futuras discussões e análise da algaroba como um indicador de degradação em áreas com características ambientais semelhantes a dos municípios estudados.

Este trabalho se insere no âmbito de vários estudos que estão sendo desenvolvidos no Grupo de Pesquisa Natureza Sociedade e Ordenamento Territorial (GEONAT) vinculado ao CNPQ que tem direcionado esforços sucessivos na construção do conhecimento de natureza socioambiental.

\section{Agradecimentos}

À FAPESB pelo apoio financeiro à pesquisa.

\section{Referências bibliográficas}

AB’SABER, A. N. Os domínios de natureza no Brasil: potencialidades paisagísticas. Cotia: Ateliê, 2003.

GISP - Programa Global de Espécies Invasoras. América do Sul invadida. A crescente ameaça das espécies exóticas invasoras. 80p, 2005.

GOMES, P. A. A algarobeira. Rio de Janeiro: Serviço de Informação Agrícola, 1961.

HUETE A.; DIDAN K.; MIURA T.; RODRIGUEZ P.; GAO X.; FERREIRA L.G. Overview of the radiometric and biophysical performance of the MODIS vegetation indices. Remote Sensing of Environment, v. 83, p. 195-213, 2002.

JÖNSSON, P.; EKLUNDH, L. TIMESAT: a program for analyzing time-series of satellite sensor data. Computers \& Geosciences, v. 30, n. 1, p. 833-845, 2004.

JUSTICE, C O.; VERMOTE, E.; TOWNSHEND, J. R. G.; DEFRIES, R.; ROY, P. D.; HALL, D. K.; SALOMONSON, V.; PRIVETTE, J. L.; RIGGS, G.; STRAHLER, A.; LUCHT, W.; MYNENI, R. B.; KNYAZIKHIN, Y.; RUNNING, S. W.; NEMANI, R. R.; WAN, Z.; HUETE, A. R.; LEEUWEN, W. V.; WOLFE, R. E.; GIGLIO, L.; MUlleR, J. P.; LEWIS, P.; BARNSLEY, M. The Moderate Resolution Imaging Spectroradiometer (MODIS): land remote sensing for global change research. IEEE Transactions on Geoscience and Remote Sensing, v. 36, n. 4, p.1228-1247, 1998.

KRUSE, F. A.; LEFKOFF, A. B.; BOARDMAN, J. B.; HEIDEBRECHT, K. B.; SHAPIRO, A. T.; BARLOON, P. J.; GOETZ, A. F. H. The Spectral Image Processing Systems (SIPS) -interactive visualization and analysis of imaging spectrometer data. Remote Sensing of Environment, v. 44, p. 145-163, 1993. 
LANDIS, J. R.; KOCH, G. G. The measurement of observer agreement for categorical data. Biometrics, v.33(1), p.159-174, 1977.t

LEÃO, T. C. C.; ALMEIDA, W. R.; DEChOUM, M. S.; ZLLER, S. R. Espécies Exóticas Invasoras no Nordeste do Brasil: Contextualização, Manejo e Políticas Públicas. Centro de Pesquisas Ambientais do Nordeste e Instituto Hórus de Desenvolvimento e Conservação Ambiental - Recife, PE. 99 p., 2011.

LIMA, P. C. F.; LIMA, J. L. S.; LIMA, A. Q. Regeneração natural em área degradada por mineração de cobre, no semi-árido brasileiro. In: Anais do $53^{\circ}$ Congresso Nacional de Botânica e da $25^{\mathrm{a}}$ Reunião Nordestina de Botânica, p. 377, UFRPE e UFPE, Recife, 2002.

LINS E SILVA, A. C. B. Characteristics of Prosopis juliflora invasion of semi-arid habitats in Norteast Brazil. Thesis (M.Sc.). University of Durham, Durham. 76 p. 1997.

OLIVEIRA, F. P., FILHO, E. I., SOARES, V. P., \& SOUZA, A. L.. Mapeamento de fragmentos florestais com monodominância de aroeira a partir da classificação supervisionada de imagens Rapideye. Scielo, $p$. 11. Acesso em 8 de novembro de 2015, disponível em http://www.scielo.br/scielo.php?script=sci_arttext\&pid=S0100- 67622013000100016\&lang=pt.

PEGADO, C. M. A.; ANDRADE, L. A.; FÉLIX, L. P.; PEREIRA, I. M. Efeitos da invasão biológica de algaroba - Prosopis juliflora (Sw.) DC. Sobre a composição e a estrutura do estrato arbustivo-arbóreo da caatinga no Município de Monteiro, PB, Brasil. Acta Botânica Brasílica, v. 20, n. 4, p. 887-898, 2006.

SAMPAIO, C. S. Abordagem metodológica baseada nos dados multitemporais MODIS EVI/NDVI para classificação da cobertura vegetal na região do Parque Nacional da Chapada dos Veadeiros/GO. 73 p. Dissertação (Mestrado em Geografia) - Universidade de Brasília, Brasília. 2007.

SEIA, 2014. Programa de Ação Estadual de Combate à Desertificação e Mitigação dos Efeitos da Seca (PAE-Bahia). Disponível em: $<$ http://www.seia.ba.gov.br/planos - programas/programa-estadual-decombatedesertifica-o-e-mitiga-o-dos-efeitos-da-seca> Acesso: 10 junho 2016.

SOARES, J. V.; BATISTA, G. T.; SHIMABUKURO, Y. E. Histórico e Descrição. In: RUDORFF, B. F. T.; SHIMABUKURO, Y. E.; CEBALlOS, J. C. (Coord.). O Sensor MODIS e suas Aplicações Ambientais no Brasil. São José dos Campos: Parêntese, 2007, 425p.

SUPERINTENDÊNCIA DE ESTUDOS ECONÔMICOS E SOCIAIS DA BAHIA (SEI). Balanço hídrico do estado da Bahia. Salvador: SEI, 1999. 